\title{
ON THE CLASSIFICATION OF SOCALLED NASAL PROPER DISEASES BASED ON A GENERAL CONSIDERATION OF NASAL CAVITY FINDINGS
}

By

R. TAKAHASHI.

\author{
From the Oto-rhino-laryngological Department, Tokyo Zikeikai \\ Medical School.
}

Socalled propery nasal diseases are seldom found singly. Generally two or thrce such diseases occur in association. Hence the author tried to consider the pathological findings of the accessory cavities as a whole and to investigate their time relation and fate, without necessarily taking heed of the distinct diagnosis of each disease. Thus the author made the following distinction as basic rhinoscopic findings:

(a) In osseous structures-normal nasal cavity, septum deviation, narrow nose, atrophic nose and lateral wall abnormaity.

(b) In soft parts-normal mucous membrane, hypertrophy, oedema, polyp, atrophy and pathological secretion.

Statistic investigation on the occurrence of these basic findings in 1099 instances of socalled properly nasal diseases enabled the author to classify them into eight distinct types and 33 modifications or intermediate types:

Type I on normal type, in which the basic findings are all practically normal. .Type II or hypertrophic type, main feature of which is the hypertrophy of the mucous membrane.

Type III or narrow type, characterized by narrowness of the nose cavity.

Type IIIv or septum type, with septum deviation of high grade, especially conspicuous in the upper part, asymmetry of nasal cavties and various morbid conditions on the lateral wall.

Type IV or polyp type, in which polyp is present.

Type $\mathrm{V}$ or hypertrophic-atropic type, with hypertrophy on the upper half and trophic changes on the lower half of the nasal cavity.

Type VI or atrophic type, characterized by spacious nasal cavity and atrophied turbinate bone, though not in the degree of ozena genuina.

Type VII or ozena type, showing the state of ozena genuina.

In each of the above eight types, chronic sinuitis may or may not occur in association.

This classification simplified the entry of the case record. On the relation as well as transition among these types and the therapeutic principle for each of them, the next paper will be referred to. 